\title{
Learning about colonization when managing metapopulations under an adaptive management framework
}

\author{
Darren M. Southwell, ${ }^{1}$ Cindy E. Hauser, and Michael A. McCarthy \\ School of BioSciences, University of Melbourne, Melbourne, Victoria, 3010, Australia
}

\begin{abstract}
Adaptive management is a framework for resolving key uncertainties while managing complex ecological systems. Its use has been prominent in fisheries research and wildlife harvesting; however, its application to other areas of environmental management remains somewhat limited. Indeed, adaptive management has not been used to guide and inform metapopulation restoration, despite considerable uncertainty surrounding such actions. In this study, we determined how best to learn about the colonization rate when managing metapopulations under an adaptive management framework. We developed a mainland-island metapopulation model based on the threatened bay checkerspot butterfly (Euphydryas editha bayensis) and assessed three management approaches: adding new patches, adding area to existing patches, and doing nothing. Using stochastic dynamic programming, we found the optimal passive and active adaptive management strategies by monitoring colonization of vacant patches. Under a passive adaptive strategy, increasing patch area was best when the expected colonization rate was below a threshold; otherwise, adding new patches was optimal. Under an active adaptive strategy, it was best to add patches only when we were reasonably confident that the colonization rate was high. This research provides a framework for managing mainland-island metapopulations in the face of uncertainty while learning about the dynamics of these complex systems.
\end{abstract}

Key words: adaptive management; bay checkerspot butterfly; Bayesian statistics; colonization; decision theory; Euphydryas editha bayensis; meta population; restoration; San Francisco Bay, California, USA; stochastic dynamic programming; uncertainty

\section{INTRODUCTION}

Conservation managers often build models of ecological systems to predict the outcome of management actions (Burgman et al. 2005). While models are useful for comparing conservation measures, modeling ecological systems is notoriously difficult and predictions are usually uncertain for various reasons (Regan et al. 2002, Bormann and Kiester 2004, Burgman 2005). Firstly, model uncertainty arises because ecological systems are complex and poorly understood; a number of plausible models might represent how a system functions (Chatfield 1995). Secondly, measurement error and biases arise when data are collected to parameterize system models, leading to observation uncertainty. Thirdly, process uncertainty can result from variability in natural systems; that is, the unpredictability of natural processes. Indeed, the challenge facing conservation managers is developing tools that

Manuscript received 18 December 2014; revised 14 April 2015; accepted 20 April 2015. Corresponding Editor: F. A. Johnson.

${ }^{1}$ E-mail: darren_southwell@hotmail.com allow for model-based decision-making while explicitly accounting for these forms of uncertainty. Failing to account for uncertainty in model-based decision-making can lead to unreliable predictions and subsequent misallocation of resources and ineffective conservation actions (Ludwig et al. 1993).

Adaptive management can help deal with uncertainty in conservation decision-making (McCarthy and Possingham 2007). The purpose of adaptive management is not only to achieve some pre-specified management objective but also to manage with the aim of learning about uncertainties that are resolvable and impact on management decisions (Holling 1978, Walters 1986, Williams 1996). In essence, adaptive management is a repetitive process of acting and reducing uncertainty by observing the outcome of those actions. Learning can be achieved in two ways: (1) under a passive adaptive strategy, by observing the outcome of past actions, or (2) under an active adaptive strategy, where managers proactively design management to deliberately accelerate the learning process. Passive adaptive management is considered precautionary because management decisions are made in response 
to what was learned in the past with only the management objective in mind (Walters 1986, McCarthy and Possingham 2007). In contrast, active adaptive management is considered experimental because a strategy may be chosen initially that is suboptimal in the short term, but the sacrifice in management improves learning such that higher returns are achieved in the long run (Walters and Hilborn 1976, Holling 1978).

Adaptive management has been applied widely to many areas of environmental management, most notably to fisheries research (Walters and Hilborn 1976, Smith and Walters 1981, Walters 1986) and wildlife harvesting in North America (Nichols et al. 1995, Williams and Johnson 1995). In most cases, learning is conducted passively rather than actively by observing the response of systems to past management actions (Parma et al. 1998). Applications of active adaptive management of environmental systems exist, but have been largely theoretical, and learning has generally focused on resolving uncertainty in a single parameter within a system model (Walters et al. 1992, Shea et al. 2002, Gerber et al. 2005, McCarthy and Possingham 2007, Hauser and Possingham 2008, Rout et al. 2009). Other examples of adaptive management include the optimal management of threatened species (Rout et al. 2009, McDonald-Madden et al. 2010), the optimal catch rate of a harvested species (Smith and Walters 1981, Nichols et al. 1995, Williams and Johnson 1995, Runge and Johnson 2002, Hauser and Possingham 2008), and vegetation restoration strategies (McCarthy and Possingham 2007, Moore and McCarthy 2010). Despite increased applications of adaptive management to environmental systems, we know of no cases in the literature where adaptive management has guided metapopulation restoration.

The optimal management of metapopulations is an important area of research in conservation biology. Metapopulations, which consist of distinct populations subject to extinction and connected by colonization (Levins 1969), are becoming increasingly prevalent in disturbed environments due to habitat loss and fragmentation. Many threatened species, in particular, are often found in isolated patches of remnant habitat and require urgent management to reduce the risk of extinction. To offset the impact of fragmentation and improve metapopulation persistence, various restoration strategies can be implemented, such as creating new patches (Westphal et al. 2003), reintroducing a species to a suitable but empty patch (Possingham 1996), protecting areas of suitable habitat (Ross et al. 2008), and modifying the spatial arrangement of patches (Moilanen and Cabeza 2002, Schultz and Crone 2005, Isaak et al. 2007). Given the rate and scale of habitat fragmentation around the globe, decision-makers usually must urgently decide which of these strategies to implement within budgetary constraints.

Metapopulation models are useful tools to help decide which management action might best meet a pre-specified management objective. Predicting the persistence of metapopulations is commonly achieved by modeling two processes: the rate at which occupied patches become extinct and the rate at which vacant patches are colonized by neighboring subpopulations (Levins 1969). A common assumption made during metapopulation modeling is that model parameters are known with certainty. In reality, model parameters will often be poorly understood when management decisions are required, making it difficult to decide between a set of management actions. The colonization rate, in particular, is notoriously difficult to measure in natural systems (Frisch et al. 2012). Given that metapopulation management is usually highly sensitive to model parameters (Nicol and Possingham 2010), managers are faced with a difficult decision in the presence of uncertainty: should a strategy be chosen despite uncertainty in model parameters or should management be delayed until these parameters are better understood? Delaying conservation action comes at the cost of potentially acting too late, whereas managing in the face of uncertainty runs the risk of choosing a suboptimal action, resulting in potentially wasted resources and suboptimal conservation outcomes (Grantham et al. 2009). So the question is: can managers learn about metapopulation parameters while managing and is it worth actively designing management to accelerate learning?

The aim of this research was to determine the optimal management strategy for a metapopulation while resolving uncertainty about metapopulation dynamics using an adaptive management framework. More specifically, we addressed the question: what is the value of learning about colonization when managing a metapopulation and what is the best way to resolve uncertainty in this parameter while managing the system? To explore these questions, we developed a metapopulation model and found the optimal passive and active adaptive management strategy for a threatened invertebrate, the bay checkerspot butterfly (Euphydryas editha bayensis). We used decision theory to determine when it is best to actively resolve uncertainty about the colonization rate in order to achieve a management objective. Decision theory is a formal approach to decision-making that involves acknowledging uncertainty and clearly specifying management objectives (Maguire 1986, Possingham et al. 2001). We established a metapopulation model within such a framework, enabling optimal decisions to be made about which management action to implement while learning about the colonization rate.

\section{Method}

\section{Formulating the problem}

We assumed that a species occupied suitable habitat patches that could either be occupied or empty. Habitat patches formed a metapopulation with an island-mainland structure (Harrison et al. 1988), containing a source 
patch that was immune to extinction and a number of island patches that were subject to extinction and colonization events. Given this structure, we assumed no migration was possible between island patches. The extinction rate of the island patches was a function of patch area and was known, but limited information was available on the colonization rate from the source patch. We tested two management objectives: (1) maximize the probability that at least one island patch is occupied at the end of the management program and (2) maximize the number of island patches occupied at the end of the management program. At any time step, managers could either do nothing $(q=0)$, increase area of existing patches $(q=1)$, or create a new patch $(q=2)$. Increasing the area of existing patches reduced the extinction rate of occupied patches, whereas adding a patch to the metapopulation provided an additional opportunity for colonization. The choice of whether to invest in creating new patches or increasing the area of existing patches depended on the colonization rate from the source patch, which was uncertain. The only way to learn about the colonization rate was to monitor unoccupied patches. In this way, adding a new patch could be treated as an experiment that created an extra opportunity to observe colonization and allow us to ask: how much effort should be put into learning about the colonization rate by adding patches to the metapopulation as opposed to adding area or doing nothing?

\section{Stochastic patch mainland-island occupancy model}

We developed a spatially implicit stochastic patch occupancy model (Ovaskainen and Hanski 2004) with a mainland-island structure (Harrison et al. 1988). We treated the mainland patch as an external colonization force and modeled the occupancy status of island patches, which are hereafter simply referred to as patches. To simplify the model, we assumed that all patches were of equal area at a given time. The state of the metapopulation was characterized by three state variables: the number of patches in the network $(M) \in\left\{M_{\min }\right.$, $\left.M_{\min }+1, \ldots, M_{\max }-1, M_{\max }\right\}$, the number of occupied patches in the network $(n) \in\{0,1,2, \ldots, M\}$, and the area of the patches in the network $(A) \in\left\{A_{\min }\right.$, $\left.A_{\min }+k, \ldots, A_{\max }-k, A_{\max }\right\}$ (Nicol and Possingham 2010). We adopted the approach presented by Nicol and Possingham (2010) and divided the state variable $A$ into discrete states of resolution $k$, ranging from a starting area $A_{\min }$ up to $A_{\max }$ and used the notation $(M, n, A)$ to refer to a metapopulation with $M$ patches each of area $A$, of which $n$ patches were occupied. Three management actions were considered: adding a new patch, adding area to each of the existing patches, and doing nothing. All modeling and optimization was conducted in R (R Development Core Team 2014). R scripts are provided in the Supplement. The probability that the metapopulation transitioned from one state to another under the alternative actions is described in the following section.

\section{State transition}

\section{Do nothing}

Transition probabilities under the do nothing strategy were modeled by a discrete-time Markov chain that described the processes of colonization and extinction. We assumed that the order of events in a time step was first colonization and then extinction (Day and Possingham 1995, Westphal et al. 2003, Nicol and Possingham 2010) and that these events were independent; that is, there was no rescue effect. We assumed binomial probability distributions for the number of colonization and extinction events (Nicol and Possingham 2010). Hence, the probability of $x$ extinction events was

$$
P[(M, n, A) \rightarrow(M, n-x, A)]=\left(\begin{array}{c}
n \\
x
\end{array}\right) e^{x}(1-e)^{n-x}
$$

where the probability of local extinction is given by $e$. The relationship between local extinction and patch area was defined as follows (Gilpin and Diamond 1976, Hanski 1994):

$$
e(M, n, A)=\frac{b}{A^{a}}
$$

where the parameters, $a$ and $b$, described the dependence of extinction probability on patch area. Further, the number of colonization events $(y)$ was modeled as

$$
P[(M, n, A) \rightarrow(M, n+y, A)]=\left(\begin{array}{c}
M-n \\
y
\end{array}\right) c^{y}(1-c)^{M-n-y}
$$

where $c$ is the probability of an island patch being colonized from the mainland patch.

\section{Adding patches}

Increasing the number of patches in the metapopulation added a new patch to the network deterministically (i.e., with probability 1 ) unless the maximum allowable number of patches $M_{\max }$ was exceeded, in which case this management option had no effect. Patches added to the metapopulation were assumed to start off vacant, and then be subject to both colonization and extinction. Transition probabilities for the metapopulation when a patch was added to the network (not including colonization and extinction) were written as

$$
\begin{gathered}
P[(M, n, A) \rightarrow(M+1, n, A)]=1 \text { for } \\
M \in\left\{M_{\min }, M_{\min }+1, \ldots, M_{\max }-1\right\} \\
P\left[\left(M_{\max }, n, A\right) \rightarrow\left(M_{\max }, n, A\right)\right]=1 .
\end{gathered}
$$

All other transition probabilities were zero when a patch was added. 


\section{Adding area}

To standardize costs, we assumed that increasing patch areas added the same area to the metapopulation as if a new patch was created (see Nicol and Possingham 2010). Therefore, if the metapopulation is in state $(M, n, A)$, the area added by increasing patch area was equal to $A / M$. However, because $A$ is a discrete variable and $A / M$ is continuous, we followed the approach outlined by Nicol and Possingham (2010) and divided the area state into bins with interval $k$. Instead of rounding to the closest area $A$, we used proximity to the closest intervals as weighting factors, which acted like transition probabilities. For example, if the metapopulation is in the state $(2,1,3)$, then 1.5 is added to the area of patches $(A / M)$. Rather than round this value, we took a linear combination of the two neighboring allowable values of $A$, meaning the metapopulation transitioned to state $(2,1,3)$ with probability $w$ (in this case being 0.5 ) and to state $(2,1,4)$ with probability $1-w$. Therefore, if $A_{\text {new }}$ is less than $A_{\text {max }}$, an increase in patch area is given by

$$
\begin{gathered}
P\left[(M, n, A) \rightarrow\left(M, n, A_{\text {new }}-\operatorname{rem}\left(\frac{A_{\text {new }}}{k}\right)\right)\right] \\
\quad=1-\frac{\operatorname{rem}\left(\frac{A_{\text {new }}}{k}\right)}{k} \\
P\left[(M, n, A) \rightarrow\left(M, n, A_{\text {new }}-\operatorname{rem}\left(\frac{A_{\text {new }}}{k}\right)+k\right)\right] \\
=\frac{\operatorname{rem}\left(\frac{A_{\text {new }}}{k}\right)}{k}
\end{gathered}
$$

where rem denotes the remainder after division. If $A_{\text {new }} \geq A_{\text {max }}$ then $P\left[(M, n, A) \rightarrow\left(M, n, A_{\text {max }}\right)\right]=1$.

To create a transition matrix element for a given strategy $q$, we calculated the probability of $y$ colonization and $x$ extinction events

$T_{q}\left[(M, n, A) \rightarrow\left(M^{\prime}, n^{\prime}, A^{\prime}\right)\right]=P_{q}\left[(M, n, A) \rightarrow\left(M_{q}, n+y-x, A_{q}\right)\right]$

where $y \in\{0,1, \ldots, M-n\}$, and $x \in\{0,1, \ldots, n+y)$. Because we assumed a mainland-island metapopulation where colonists disperse from a mainland patch, island patches could be colonized even if none were occupied. Since the colonization and extinction events were assumed to obey the Markov property of independent events, the transition probabilities were written as

$$
\begin{aligned}
T_{q} & {\left[(M, n, A) \rightarrow\left(M^{\prime}, n^{\prime}, A^{\prime}\right)\right]=P_{q}\left[(M, n, A) \rightarrow\left(M_{q}, n+y, A_{q}\right)\right] } \\
& \times P_{q}\left[(M, n+y, A) \rightarrow\left(M_{q}, n+y-x, A_{q}\right)\right] .
\end{aligned}
$$

\section{Uncertainty in the colonization parameter}

Rather than set the colonization rate as a fixed value, we modeled uncertainty in this parameter with a beta probability distribution. By choosing this distribution, we considered that all possible colonization rates between 0 and 1 were plausible, given by

$$
f(c ; \alpha, \beta)=\frac{1}{B(\alpha, \beta)} c^{\alpha-1}(1-c)^{\beta-1}
$$

where $B(\alpha, \beta)$ is the beta function and $\alpha$ and $\beta$ are parameters of the probability distribution. Different states of belief in the colonization rate $c$ can be represented by varying the values of $\alpha$ and $\beta$. The expected colonization rate is given by the mean of the beta distribution $E(c)=\alpha /(\alpha+\beta)$ and the sum of $\alpha+\beta$ can be interpreted as our confidence in this estimate. As the sum of $\alpha$ and $\beta$ increases, we become more confident in our estimate of the colonization rate. Thus, $\alpha$ and $\beta$ provide sufficient information to completely describe belief and confidence in the colonization rate at any time during the management program.

\section{Updating belief in the colonization rate}

We used Bayesian updating to describe how observing the turnover rate of vacant patches improved the estimate of the colonization rate. When a prior has a beta distribution with parameters $\alpha$ and $\beta$ and there are $s$ monitored successes and $f$ monitored failures from $s+f$ independent trials (i.e., binomial sampling), the posterior will be a beta distribution with parameters $\alpha+s$ and $\beta+f$. In our case, we considered possible colonization of each vacant patch as an independent trial and updated our belief in the colonization rate by monitoring the number of vacant patches that became occupied (assuming a detection probability of 1). At any point in time, the number of independent trials was equal to the number of unoccupied patches $(M-n)$. When $y$ of the vacant $(M-n)$ patches were colonized, the first parameter $(\alpha)$ was increased by $y$ and the second parameter $(\beta)$ increased by $M-n-y$. Consequently the number of colonization events $y$ in the face of this uncertainty follows a beta-binomial distribution

$$
\begin{aligned}
& P_{q}\left[(M, n, A) \rightarrow\left(M_{q}, n+y, A_{q}\right)\right]= \\
& \quad\left(\begin{array}{c}
M-n \\
y
\end{array}\right) \frac{B(\alpha+y, \beta+M-n-y)}{B(\alpha, \beta)}
\end{aligned}
$$

where, if we assume a beta $\left(\alpha_{0}, \beta_{0}\right)$ distribution for $c$ at the beginning of management, $\alpha-\alpha_{0}$ is the number of previous successful colonization events and $\beta-\beta_{0}$ is the number of previous unsuccessful colonization events. Assuming there is at least one vacant patch, we can learn about the metapopulation under all of the management actions; however, adding a patch to 
the network creates an additional independent trial in which to observe colonization and accelerate the learning process.

\section{Finding the optimal management strategy}

We used stochastic dynamic programming (SDP) to determine the optimal management decision for each state of the metapopulation at each point in time. SDP can be applied where sequential management decisions are made to stochastic systems with a finite number of states (Bellman 1957, Lubow 1996, Mangel and Clark 2000, Marescot et al. 2013). After discretizing the system into states, the first step of an SDP is to define a management objective. We ran the model under two different management objectives, firstly, to maximize the probability that at least one of the island patches was occupied at the end of a management program $\left(t_{\text {max }}\right)$. The terminal reward can be written as

$$
V_{1}\left((M, n, A, \alpha, \beta), t_{\max }\right)=\left\{\begin{array}{cc}
1 & \text { if } n>0 \\
0 & \text { otherwise }
\end{array}\right.
$$

where $V_{1}((M, n, A, \alpha, \beta), t)$ is the probability that there are one or more occupied patches at time $t_{\max }$ if the current state of the system is $(M, n, A, \alpha, \beta)$ at time $t$ and we act optimally until $t_{\max }$. By selecting this value function, the number of patches occupied at the end of the management program is not considered important, provided at least one of the island patches is occupied. Secondly, we found the optimal management strategy with the management objective of maximizing the number of patches occupied at $t_{\max }$, with the terminal reward

$$
V_{2}\left((M, n, A, \alpha, \beta), t_{\max }\right)=n
$$

where $V_{2}((M, n, A, \alpha, \beta), t)$ is the expected number of patches occupied at time $t_{\max }$ given the system is in state $(M, n, A, \alpha, \beta)$ at time $t$ and the metapopulation is managed optimally from time $t$ to $t_{\max }$. This objective differs from the first objective because managers seek to maximize the number of patches occupied at the end of the management program, rather than just ensuring at least one is occupied. SDP works by calculating the final reward for each state at the terminal time $\left(t_{\max }\right)$, based on the objective function. The SDP iteratively steps backward in time to evaluate each possible system state $(M, n, A)$ and belief state $(\alpha, \beta)$ in the previous time step, using transition probabilities given by the metapopulation model. Of all the possible management actions, the SDP selects the one that results in the highest expected return for each system state at each point in time. We assumed the management program ran for $10 \mathrm{yr}\left(t_{\max }=10\right)$ with each time step corresponding to $1 \mathrm{yr}$. Our choice of time horizon was limited by the number of states the metapopulation could be in as SDP is often subject to the "curse of dimensionality" (Bellman 1957). We selected a time horizon that was computationally feasible. However, 10 yrs is also a reasonable time horizon to assess performance of conservation management actions. The SDP was run under two learning strategies: passive and active adaptive management.

\section{Passive adaptive management}

Under passive adaptive learning, we updated our belief in the colonization rate by monitoring the proportion of vacant patches that were colonized $(y)$ up until the current time step $t$ of a management program. The optimal passive adaptive strategy was found by searching through all possible states $(M, n, A, \alpha, \beta)$ at each point in time, starting at the terminal time $\left(t_{\max }\right)$ and working backward. For each state and time, the SDP calculated the probability of transitioning to all possible future states at time $t+1$. Transition probabilities to future states depended on the management action $q$ and belief in the expected colonization rate $(\alpha, \beta)$. The value of each action $V$ at time $t$ was calculated as the weighted sum of the value of transitioning to future states at time $t+1$ multiplied by the probability of transitioning to those states. The process was repeated for each action $q$, in each state, with the SDP returning the action that maximized the management objectives for each state. This process was described by the recursive dynamic programming equation

$$
\begin{aligned}
& V_{q}((M, n, A, \alpha, \beta), t)=\max _{q \in(0,1,2)} \\
& {\left[\sum_{m^{\prime}=M_{\min }}^{M_{\max }} \sum_{n^{\prime}=0}^{M} \sum_{A^{\prime}=A}^{A_{\max }} \sum_{y=0}^{M-n} P_{q}\left[(M, n, A) \rightarrow\left(M^{\prime}, n^{\prime}, A^{\prime} \mid \alpha, \beta\right)\right] \times\right.} \\
& \left.V_{q}\left(\left(M^{\prime}, n^{\prime}, A^{\prime}, \alpha, \beta\right), t+1\right)\right]
\end{aligned}
$$

where $V$ is the value of the optimal action $(q)$ under passive adaptive management, depending on the current time step in the restoration project $(t)$, current knowledge about the colonization rate (a beta distribution with parameters $\alpha$ and $\beta$ ), the number of patches $(M)$, the number of occupied patches $(n)$, and the area of patches $(A)$. Therefore, when finding the optimal passive adaptive strategy, the SDP did not anticipate any further resolution of uncertainty in the colonization rate but incorporated what was learned by monitoring all possible outcomes of past actions.

\section{Active adaptive management}

The SDP performed the same routine for active adaptive management as for passive adaptive management; the optimal strategy was found for each state of the system at each point in time. However, under active adaptive management, possible changes to $\alpha$ and 
$\beta$ in the future were incorporated into the dynamic programming equation. Transition probabilities to possible future states at time $t+1$ were still calculated using $\alpha$ and $\beta$, but the value of being in these future states included the learning potential of each action. Hence $\alpha$ and $\beta$ were updated with new information that will be gained through management, $(\alpha+s)$ and $\left(\beta+m^{\prime}-n-s\right)$ respectively. Thus, the recursive dynamic programming equation for active adaptive management is

$$
\begin{aligned}
& V_{q}((M, n, A, \alpha, \beta), t)=\max _{q \in(0,1,2)} \\
& \quad\left[\sum _ { M ^ { \prime } = M _ { \operatorname { m i n } } } ^ { M _ { \operatorname { m a x } } } \sum _ { n ^ { \prime } = 0 } ^ { M } \sum _ { A ^ { \prime } = A } ^ { A _ { \operatorname { m a x } } } \sum _ { y = 0 } ^ { M - n } P _ { q } \left[(M, n, A) \rightarrow\left(M^{\prime}, n^{\prime}, A^{\prime} \mid \alpha, \beta\right) \times\right.\right. \\
& \left.\left.\quad V_{q}\left(\left(M^{\prime}, n^{\prime}, A^{\prime}, \alpha+y, \beta+M^{\prime}-n-y\right), t+1\right)\right]\right] .
\end{aligned}
$$

\section{Case study}

To demonstrate our framework specifically, we found the optimal passive and adaptive management strategy for a threatened invertebrate, the bay checkerspot butterfly (Euphydryas editha bayensis). E. editha bayensis is a medium-sized butterfly endemic to the San Francisco Bay region of California, USA (Ehrlich et al. 1975), protected since 1987 under the United States Endangered Species Act (United States Federal Register 1987). It is restricted to native grasslands in highly discrete local populations (Ehrlich 1961), where extinction, dispersal, and colonization have allowed for longterm persistence (Murphy 1984, Harrison et al. 1988). This pattern of occupancy resembles a mainland-island metapopulation, consisting of a large source population in the order of $10^{6}$ adult butterflies on a 200-ha patch adjacent to 27 smaller island populations (Harrison et al. 1988). The large source population is not thought to be susceptible to extinction, but the island populations are prone to relatively frequent extinction events caused, at least in part, by habitat deterioration and drought (Ehrlich et al. 1980). The successful colonization of island patches from the source population has been well-documented (Ehrlich et al. 1975, Harrison et al. 1988), but the rate of colonization is not known with certainty (Murphy et al. 1990). Since the 1980s, populations of E. editha bayensis have declined due to the introduction of noxious weeds, drought events, overgrazing, and urbanization (Ehrlich et al. 1975).

We parameterized our mainland-island metapopulation model for E. editha bayensis and found the optimal passive and active adaptive management strategy for all possible states at each point in time using SDP. To run the SDP, we had to specify the extinction-area relationship for $E$. editha bayensis. Although factors such as topography and vegetation type have been shown to influence local extinction (Launer and Murphy 1994, Preston et al. 2012), we assumed that extinction was only a function of patch area. We estimated the extinction-area relationship for E. editha bayensis by fitting the extinction-area curve to data published in Harrison et al. (1988; Appendix). To test the sensitivity of the optimal adaptive management strategy to uncertainty in the extinction-area curve for E. editha bayensis, we also ran the SDP using the upper and lower $95 \%$ confidence intervals. We allowed for up to 10 prior observations of the turnover rate $(\alpha+\beta)$ at the start of a management program, i.e., $\alpha_{0} \in\left\{\begin{array}{llll}1, & 2, \ldots, & 11\end{array}\right\}$, $\beta_{0} \in\left\{1,2, \ldots, 11-\alpha_{0}\right\}$. The minimum $\left(A_{\min }\right)$ and maximum allowed area $\left(A_{\max }\right)$ was set to 2 and 25 ha respectively, with a resolution $k$ equal to 1 . The minimum number of patches $\left(M_{\min }\right)$ was set to two, with $M_{\max }$ set to 13 . The time horizon was set at $10 \mathrm{yr}$.

\section{Simulating management actions}

We simulated the optimal passive and adaptive management strategies for a subset of island patches for a 10-yr management program using transition probabilities generated by the SDP. For the purpose of illustration, we assumed managers could manage two of the island patches at the start of the restoration program $(M=2)$, one of which was occupied $(n=1)$. The initial starting area $(A)$ was 2 ha. At each time step, managers could either add area to existing patches, add a patch, or do nothing, with the goal of maximizing the probability of at least one patch being occupied at the end of the management program (objective 1). When running the simulations, we assumed the initial patches, and any new patches added to the metapopulation, were of equal distance to the source patch and therefore subject to a true colonization rate of 0.1 . Our prior belief in the colonization rate was represented by a beta $(1,2)$ prior distribution, which is approximately equal to published estimates of the colonization rate for patches closest to the source patch (Harrison et al. 1988).

Given these initial conditions, we simulated the effect of passive and active adaptive management on the E. editha bayensis metapopulation over $10 \mathrm{yr}$ of management. We performed 1000 replicate simulations under each strategy and averaged the number of patches occupied $(n)$, the number of learning opportunities $(\alpha+\beta)$, and belief in the colonization rate $E(c)$ over time. By simulating the optimal passive and adaptive management strategies, the potential benefits of actively learning about colonization could be compared with managing the system passively. To determine the effectiveness of learning (either passively or actively), we also simulated the probability of one patch being occupied at the end of the management program if managers were to do nothing, always add patches, or always add area to the metapopulation without learning about the colonization rate. A summary of the initial conditions used in the simulations is presented in Table 1. 
TABle 1. Parameters and initial conditions used to simulate management strategies for Euphydryas editha bayensis.

\begin{tabular}{lll}
\hline Parameter & \multicolumn{1}{c}{ Definition } & \multicolumn{1}{c}{ Value } \\
\hline$t_{\max }$ & Time horizon & $10 \mathrm{yr}$ \\
$M_{\min }$ & Minimum number of patches & 2 \\
$M_{\max }$ & Maximum number of patches & 13 \\
$A_{\min }$ & Minimum area & $2 \mathrm{ha}$ \\
$A_{\max }$ & Maximum area of patches & $25 \mathrm{ha}$ \\
$M$ & Initial number of patches & 2 \\
$n$ & Initial number of occupied patches & 1 \\
$A$ & Initial area & $2 \mathrm{ha}$ \\
$k$ & Resolution of area & $1 \mathrm{ha}$ \\
$a$ & Species area relationship & $0.34^{\dagger}$ \\
$b$ & Species area relationship & $0.38^{\dagger}$ \\
$\alpha_{0}$ & Initial alpha shape parameter & $1^{\dagger}$ \\
$\beta_{0}$ & Initial beta shape parameter & $2^{\dagger}$ \\
\hline
\end{tabular}

†Source: Harrison et al. (1988).

\section{Results}

We found the optimal management strategy for all possible states of a metapopulation $(M, n, A)$ and expected colonization rates (i.e., all combinations of $\alpha, \beta)$ at each point in time for the two different management objectives over a 10 -yr time horizon. Not surprisingly, it was always optimal to add a patch or add area to the metapopulation when the limits $A_{\max }$ or $M_{\text {max }}$ had been reached, respectively, because only one action offered potential benefits. When both $A_{\max }$ and $M_{\max }$ had been reached, managers had no option but to do nothing. Given the large number of states that the metapopulation could be in, we present results for only a selection of combinations when $A<A_{\max }$ and $M<M_{\max }$ to demonstrate general trends in management decisions over time (Figs. 1-4). The optimal management strategies are presented as a function of the expected colonization rate $E(c)=\alpha /(\alpha+\beta)$ and our confidence in this estimate, given by $\alpha+\beta$, with each possible state $(\alpha, \beta)$ marked with a symbol. This relationship between the expected colonization rate $E(c)$ and the number of observations determines the distribution of circles in Figs. 1-4. The number of possible expected colonization rates $E(c)$ increases as the number of observations $\alpha+\beta$ increases. There are no circles in the bottom left or right portions of the figures because very high and very low estimates of colonization require many observations.

\section{Passive adaptive management}

\section{General result}

Under a passive adaptive management strategy, there was a threshold (the boundary between gray shading and no shading) in the expected colonization rate, below which it was optimal to increase patch areas, and above which it was optimal to add a patch (Figs. 1-4). This threshold was insensitive to confidence in the expected colonization rate $(\alpha+\beta)$ because future learning opportunities were not valued in the dynamic recursive equation. Generally speaking, it was only best to add area to existing patches when the expected colonization rate was low, less than $\sim 0.1$ for most states of the metapopulation. Otherwise, it was always best to add patches. This result makes intuitive sense; we only add patches to the metapopulation when past experience suggests the colonization rate is sufficiently high for a new patch to be colonized.

\section{Sensitivity of area-patch threshold}

The optimal passive adaptive strategy was sensitive to both the state of the metapopulation and choice of model parameters. This meant the boundary between when it was optimal to add area or add a patch (where the gray shading ends) was state dependent. As the number of patches in the metapopulation increased, there were more belief states in which it was best to add patches (Fig. 1). In contrast, as the number of occupied patches increased, there were fewer belief states in which it was best to add patches (Fig. 2). In both cases, the optimal passive adaptive strategy was relatively insensitive to changes in area (Figs. 1, 2). As time progressed, there were fewer belief states in which it was best to add patches (Fig. 3). The optimal management strategy was sensitive to the management objective: when the objective was to maximize the number of patches occupied, it was almost always best to add patches to the metapopulation rather than add area (Fig. 3). The optimal passive adaptive strategy was sensitive to uncertainty in the extinction-area curve for $E$. editha bayensis: there were more belief states in which it was best to add area using the lower 95\% confidence interval compared to the upper $95 \%$ confidence interval (Fig. 4). This was because the lower $95 \%$ confidence interval had a lower extinction rate than the best fit or upper $95 \%$ confidence interval, and thus meant that adding area was more effective at increasing survival compared to adding patches.

\section{Learning from active adaptive management}

\section{General results}

There were more belief states in which it was optimal to add area under an active adaptive management strategy compared with a passive adaptive management strategy (shown by the solid black and open circles in Figs. 1-4). This was because the optimal active adaptive management strategy was sensitive to our confidence in the expected colonization rate. Generally speaking, the area-patch threshold (the boundary between solid black circles and open circles) under active adaptive management is similar to passive adaptive management when we were confident in our 

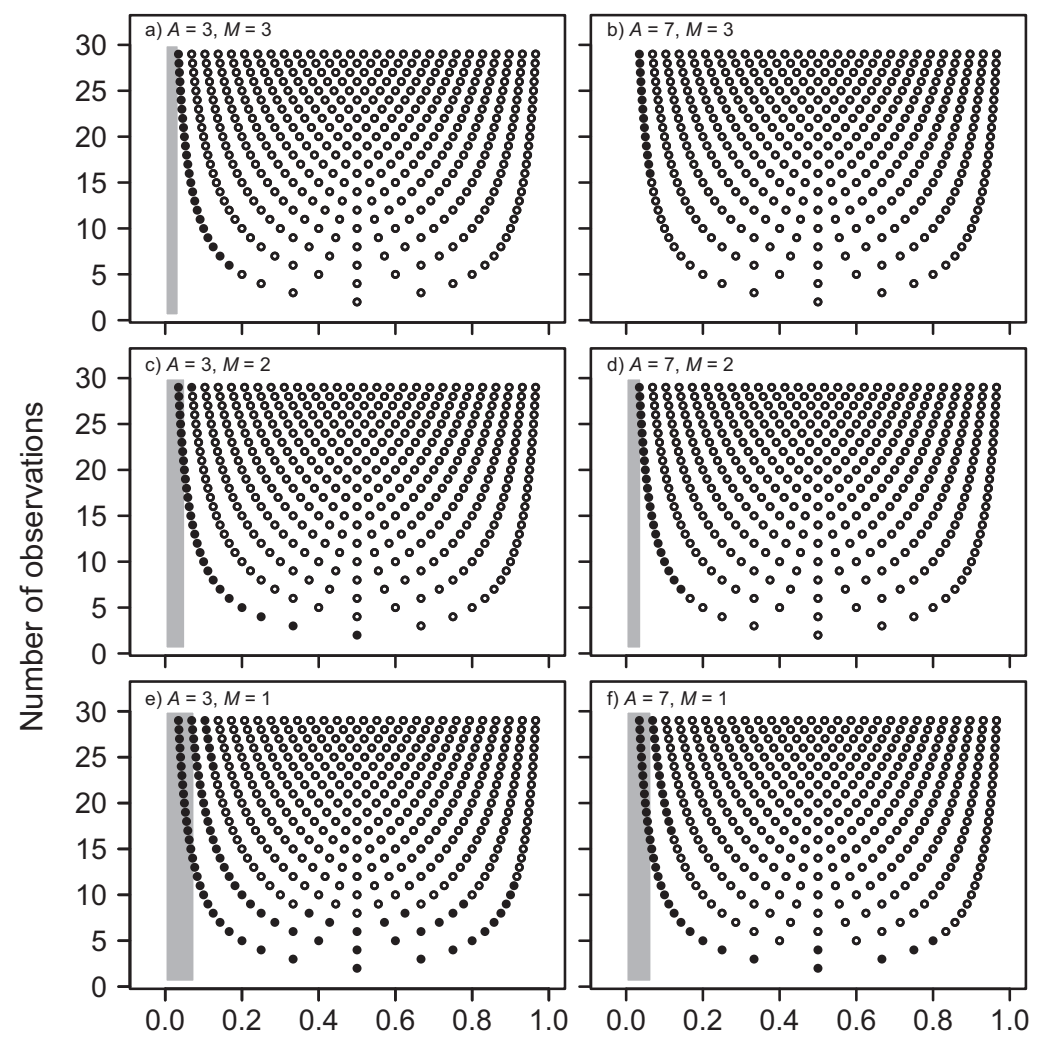

Expected colonization rate

FIG. 1. Adaptive restoration strategies in the middle of a 10-yr management program $(t=5 \mathrm{yr})$ for a metapopulation of Euphydryas editha bayensis with one occupied patch $(n=1)$ and extinction-area parameters $a=0.34$ and $b=0.38$. The results presented here are for a metapopulation with $(\mathrm{a}, \mathrm{b})$ three patches $(M=3),(\mathrm{c}, \mathrm{d})$ two patches $(M=2)$, and $(\mathrm{e}, \mathrm{f})$ one patch $(M=1)$ of (a, c, e) area $A=3$ ha and (b, d, f) $A=7$ ha in size under objective 1 (maximize the probability of at least one patch being occupied at the end of the time horizon). The expected value in the colonization rate $E(c)$ is presented on the $x$-axis and the number of observations $(\alpha+\beta)$ on the $y$-axis. Shading indicates the optimal passive adaptive management strategy; add area to patches ( $q=1$, gray shading) or add patches $(q=2$, no shading). Solid black circles represent states where it is optimal to add area to the metapopulation under active adaptive management, open circles represent where it is optimal to add patches.

estimate of the colonization rate (i.e., if we had observed the turnover rate of a vacant patch $\sim 30$ times). However, the optimal active adaptive management strategy favored adding area to existing patches when the expected colonization rate, and our confidence in this estimate, was low (Fig. 1-4). This result is rather counterintuitive: we might expect the best way to actively learn about the colonization rate is to intentionally create additional learning opportunities by adding vacant patches to the metapopulation. In this case, the active adaptive strategy favored a precautionary approach to management by taking the guaranteed returns of adding area over the uncertain benefits of adding patches.

\section{Sensitivity of area-patch threshold to model states and parameters}

The optimal active adaptive strategy was sensitive to both the state of the metapopulation and choice of model parameters (Figs. 1-4). For example, as the number of patches in the metapopulation increased and confidence in the colonization rate was low, the best strategy became less precautionary about adding patches to the metapopulation (Fig. 1). In other words, with few patches in the metapopulation and only a vague understanding of the colonization rate, it was best to learn about colonization by adding area and monitoring the turnover rate of existing vacant patches. This is because the probability of metapopulation persistence increased as the number of patches increased, so there was less to be lost through experimentation. A similar trend existed as the number of occupied patches in the metapopulation decreased (Fig. 2). There were fewer states in which to add patches as the number of occupied patches approached the number of patches in the metapopulation. When the number of vacant patches was small, creating an additional single learning opportunity did not outweigh the benefit of adding area. 

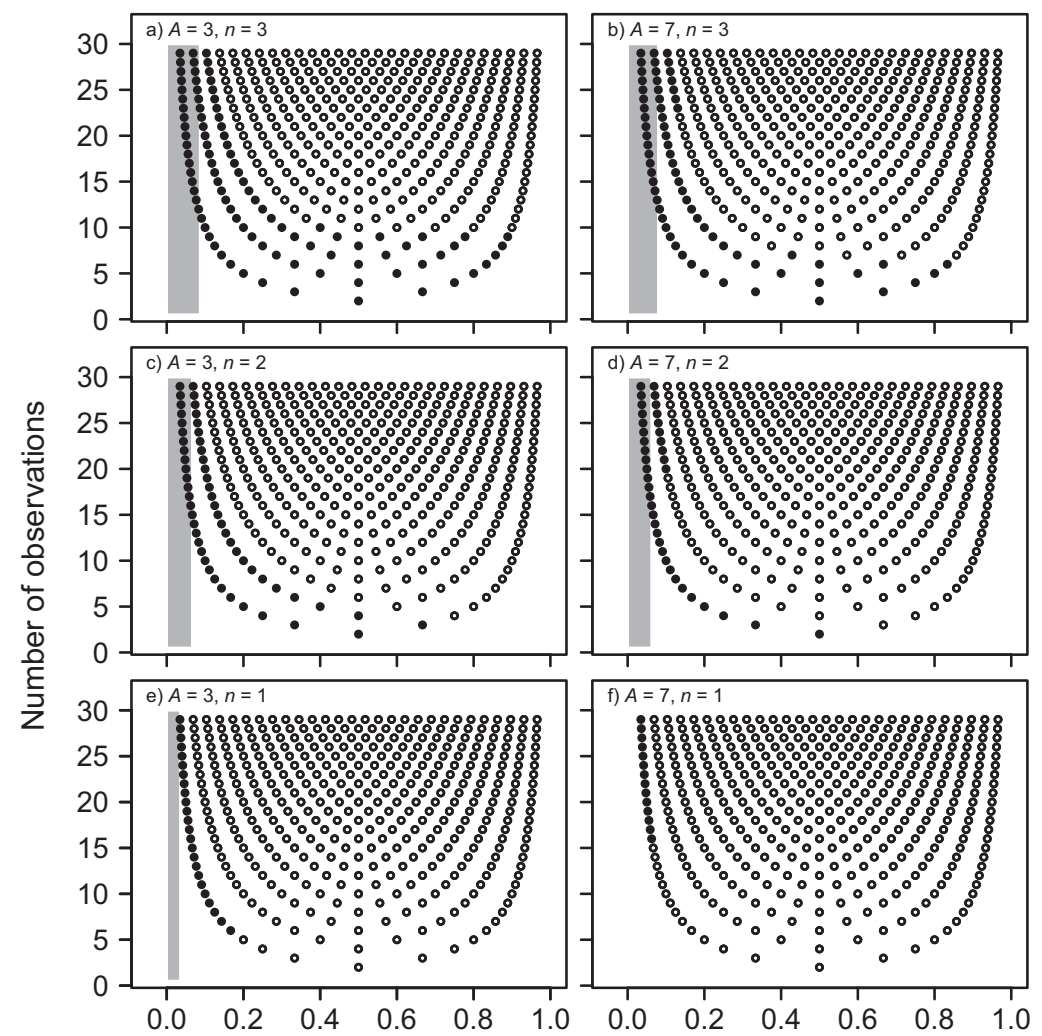

Expected colonization rate

FIG. 2. Adaptive restoration strategies in the middle of a 10 -yr management program $(t=5 \mathrm{yr})$ for a metapopulation of $E$. editha bayensis with three patches and extinction-area parameters $a=0.34$ and $b=0.38$. The results presented here are for a metapopulation with (a, b) three occupied patches $(n=3),(\mathrm{c}, \mathrm{d})$, two occupied patches $(n=2)$, and $(\mathrm{e}, \mathrm{f})$ one occupied patch $(n=1)$ of $(\mathrm{a}, \mathrm{c}, \mathrm{e}) A=3 \mathrm{ha}$ and (b, d, f) $A=7$ ha in size under objective 1 (maximize the probability of at least one patch being occupied at the end of the time horizon). Axes, $q$ values, shading, and circle types are as in Fig. 1.

\section{Sensitivity of area-patch threshold to time and management objectives}

The optimal active adaptive management strategy was sensitive to time and alternative management objectives. For both management objectives, it became more favorable to add patches to the metapopulation as time progressed (Figs. 3, 4). Generally speaking, the optimal active adaptive management strategy was to add area early in the restoration program if the colonization rate was uncertain and learn by observing the turnover rate of existing vacant patches. Patches should then be added if past experience suggested that colonization was reasonably high. Importantly, the optimal active adaptive management strategy was equal to the optimal passive adaptive management strategy in the final time step because there was no time remaining to actively learn about colonization (Fig. 3, 4). The optimal active adaptive strategy was sensitive to alternative management objectives. In general, it was more important to add patches to the metapopulation when the goal was to maximize the number of patches occupied at the end of the management program.
Under this objective, it was almost always best to add patches to the metapopulation in the final time steps.

\section{Simulating passive and active adaptive management}

There was little difference between managing the E. editha bayensis metapopulation under passive or active adaptive management (Fig. 5a). The probability of at least one patch being occupied at the end of a 10-yr management program was 0.880 under active adaptive management and 0.885 under passive adaptive management, given the initial state of the metapopulation, our prior belief about the colonization rate, and a true colonization rate of 0.1 . Adaptive management performed considerably better than consistently adding area or doing nothing. The probability of at least one patch being occupied after $10 \mathrm{yr}$ was 0.713 when area was added and 0.397 when no action was taken. Consistently adding patches to the metapopulation performed as well as passive or active adaptive management: under this strategy the 

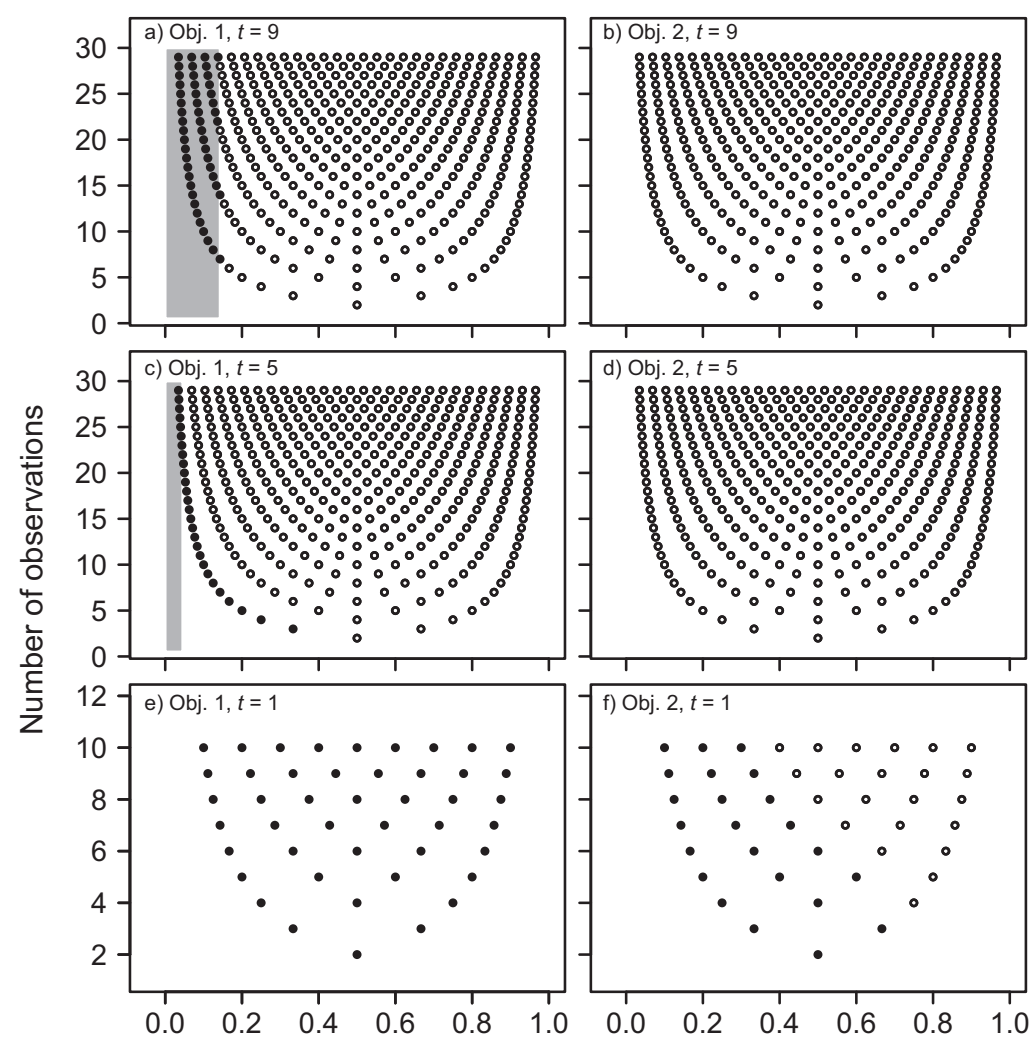

Expected colonization rate

FIG. 3. Adaptive restoration strategies for a metapopulation of E. editha bayensis with two patches. The results presented here are for objective 1 (Obj. 1; maximize the probability of at least one patch being occupied) and objective 2 (Obj. 2; maximize the number of patches occupied) at (a,b) the end of management program (time $t=10 \mathrm{yr}),(\mathrm{c}, \mathrm{d})$ five years into a management program $(t=5 \mathrm{yr}$ ), and $(\mathrm{e}, \mathrm{f})$ at the start of a management program $(t=1 \mathrm{yr})$. Patch area $A=7$ ha, one of which is occupied, with extinction-area parameters $a=0.34$ and $b=0.38$. A maximum of 10 prior observations of the colonization rate are plotted at the start of management $(\mathrm{e}, \mathrm{f})$. In the middle and end of the management program $(\mathrm{a}-\mathrm{d})$, the number of observations is truncated at 30 . Axes, $q$ values, shading, and circle types are as in Fig. 1.

probability of at least one patch being occupied at the end of the management program was 0.892 . We note that the relative performance of adding patches or adding area depends on the true value of the colonization rate. A colonization rate less than 0.1 would decrease the effectiveness of adding patches and increase the performance of just adding area to the metapopulation.

Given the initial conditions assumed in our study and a true colonization rate of 0.1 , managers had on average 32 learning opportunities under active adaptive management, 55 learning opportunities under passive adaptive management, 55 learning opportunities if patches were always added, and 14 learning opportunities if area was always added (Fig. 5b). The number of learning opportunities was higher under passive adaptive management compared with active adaptive management because passive learning favored adding patches to the metapopulation early in the management program when the colonization rate was uncertain. We could still learn about the colonization rate by doing nothing: on average there were 16 opportunities to observe if a vacant patch was colonized during the 10-yr management program. Because more patches were created under passive adaptive management, and thus there were more learning opportunities, estimates of the colonization rate were more precise under passive adaptive management. This can be seen by the narrower confidence intervals in the expected colonization rate under passive adaptive management (Fig. 5c).

\section{Discussion}

Optimizing current and future management of metapopulations is a key topic in ecology due to the rate and scale at which habitat fragmentation is occurring around the globe. Managers are often required to make decisions regarding the restoration of metapopulations with only a vague understanding of the system 

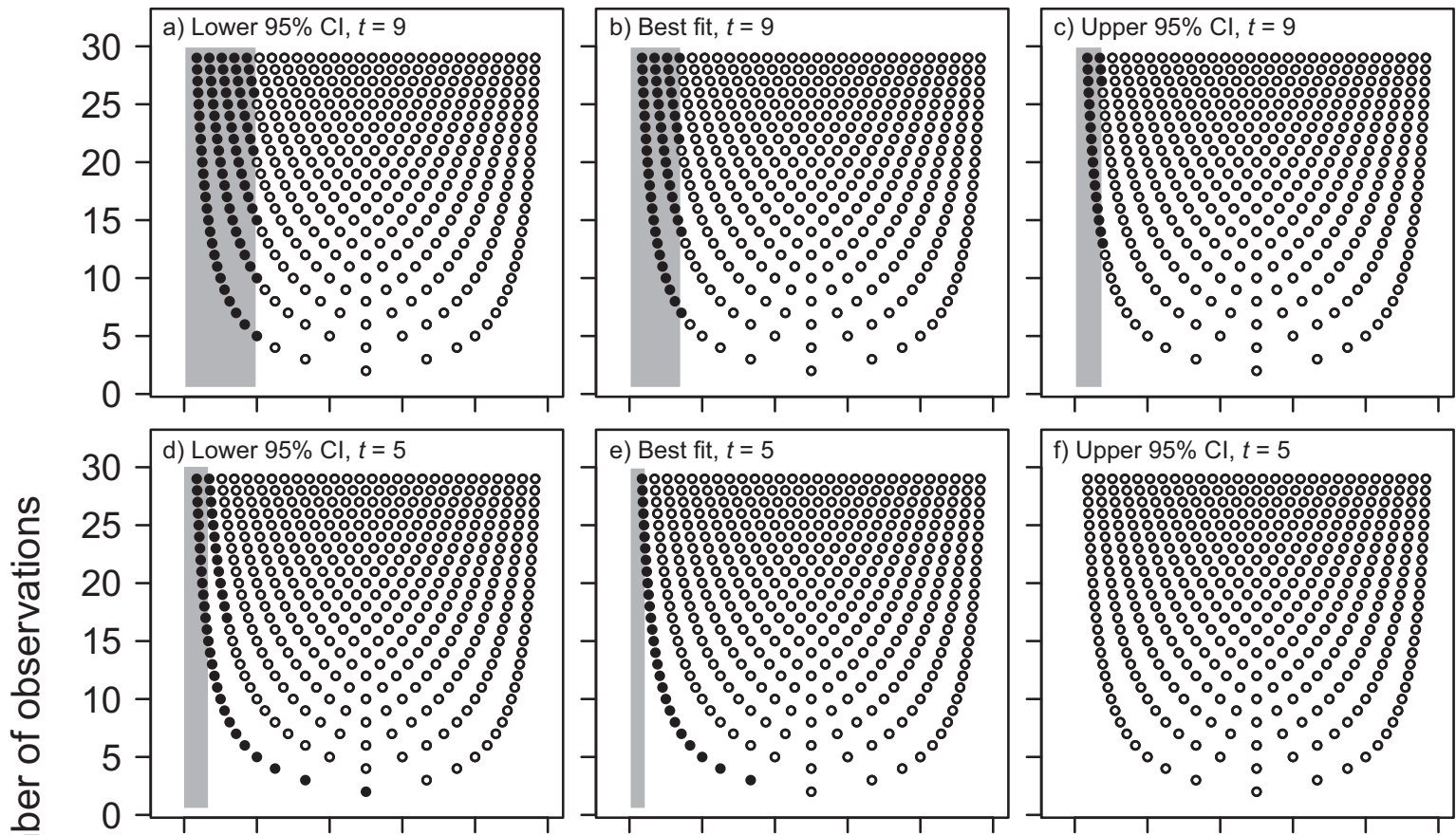

$\frac{\xi}{\mathrm{z}}$
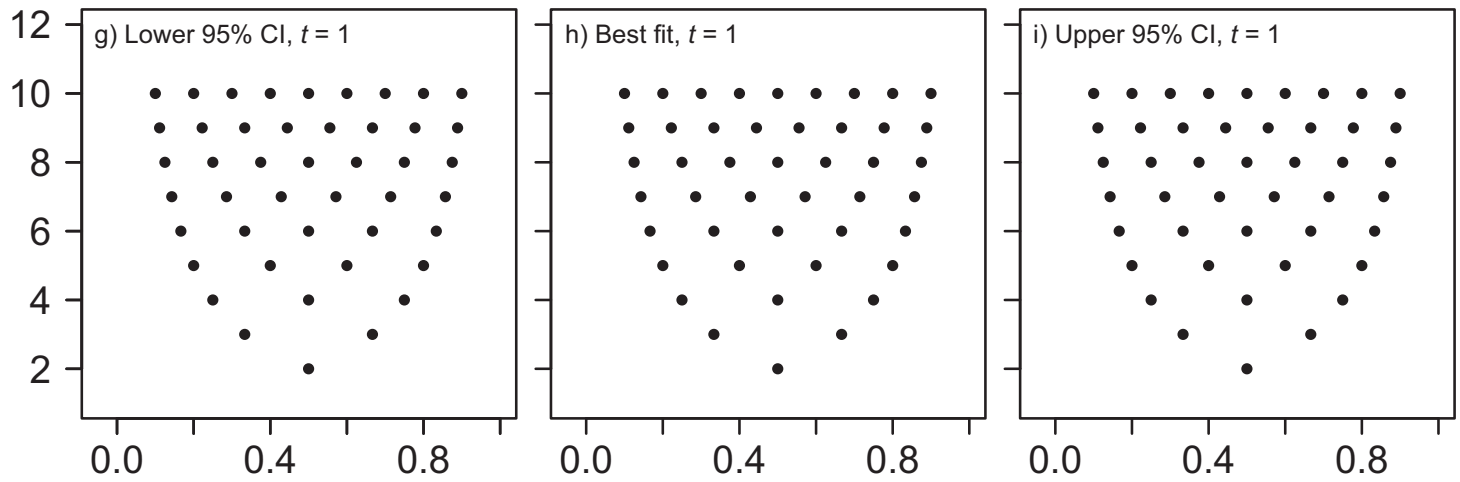

Expected colonization rate

FIG. 4. Adaptive restoration strategies for a metapopulation of $E$. editha bayensis with two patches. The results presented here are for objective 1: $(\mathrm{a}-\mathrm{c})$ at the end of management program $(t=10 \mathrm{yr}),(\mathrm{d}-\mathrm{f})$ five years into a management program $(t=5 \mathrm{yr})$, and $(\mathrm{g}-\mathrm{i})$ at the start of a management program $(t=1 \mathrm{yr})$. One of the patches is occupied $(n)$ and both are $A=5$ ha in size. The stochastic dynamic programming was run with three extinction-area curves; a best fit (middle column) and lower and upper $95 \%$ confidence intervals (left and right columns, respectively). A maximum of 10 prior observations of the colonization rate area plotted at the start of management $(\mathrm{g}-\mathrm{i})$. In the middle and end of the management program (a-f) the number of observations are truncated at 30 . Axes, $q$ values, shading, and circle types are as in Fig. 1.

dynamics (Etienne and Heesterbeek 2001). We have constructed a management strategy for species occupying mainland-island metapopulations that incorporates uncertainty in the colonization rate and allows for this uncertainty to be resolved over the course of a management program. While theoretical examples of adaptive management exist for many fields of environmental management (McCarthy and Possingham 2007, Hauser and Possingham 2008, Rout et al. 2009), to our knowledge this is the first application of the framework to the restoration of metapopulations.
Metapopulation restoration provides a good setting for adaptive management; decisions are often required urgently, there is usually a limited understanding of the dynamics driving optimal decisions, and management is usually repeated over time, allowing for opportunities to learn from past actions.

By comparing passive and active management, we determined the value of actively learning about colonization through experimentation. Our results suggest that the active adaptive approach is precautionary compared with the passive adaptive strategy, favoring 

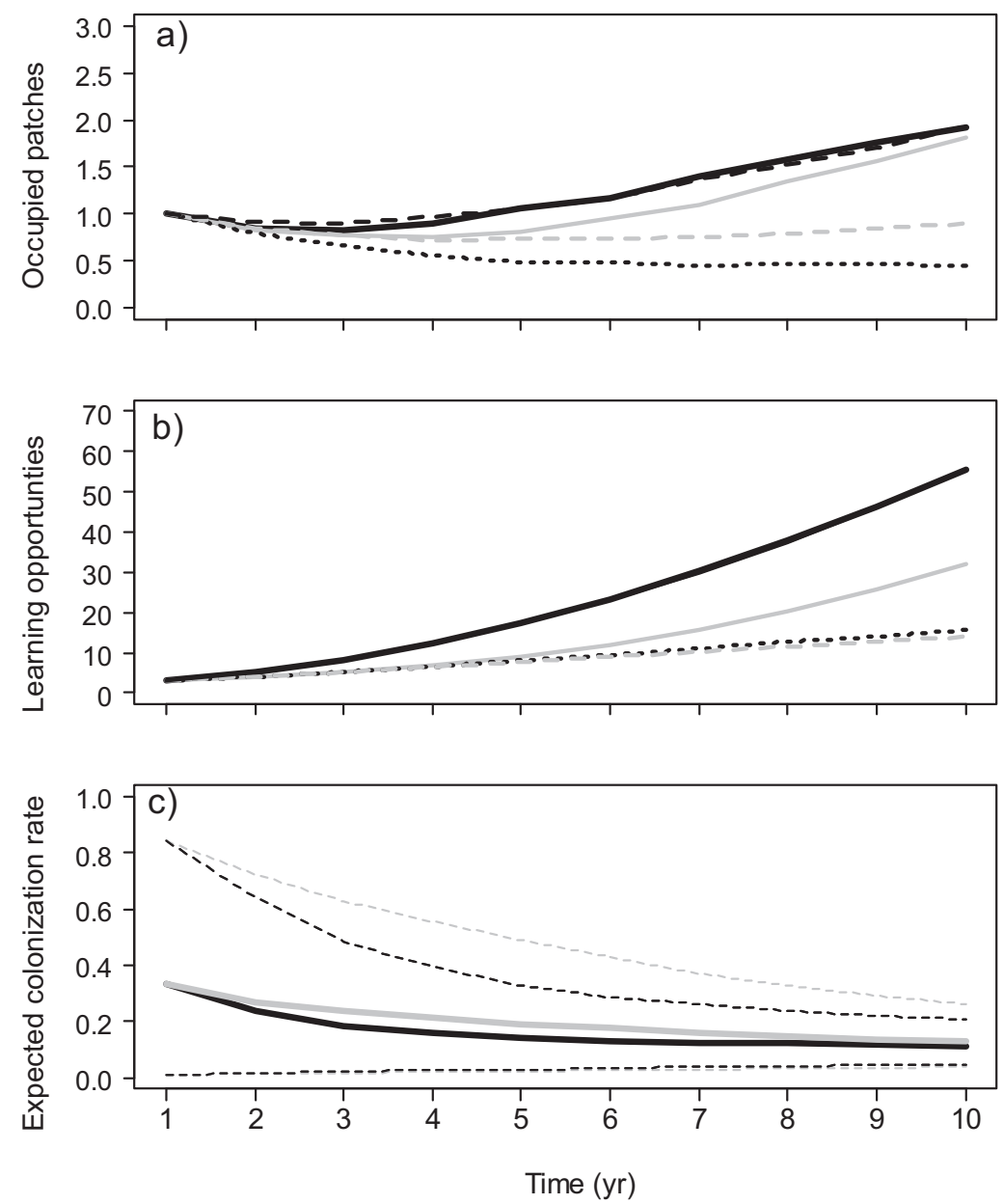

FIG. 5. (a) The number of patches occupied by E. editha bayensis over a $t_{\max }=10$-yr management program, averaged over 1000 simulations under passive adaptive management (black solid line), active adaptive management (gray solid line), adding patches (black dashed line), adding area (gray dashed line), and doing nothing (black dotted line). Initially, the metapopulation contained two patches $(M=2)$, one of which was occupied $(n=1)$, and $A=2$ ha in size. The true colonization rate was set to $c=0.1$. (b) The total number of learning opportunities $(\alpha+\beta)$ throughout the management program under passive adaptive management (black solid line), active adaptive management (gray solid line), adding patches (black dashed line), adding area (gray dashed line), and doing nothing (black dotted line). (c) Belief in the expected colonization rate $E(c)$ denoted by the mean (solid line) with 2.5 th and 97.5 th percentiles on the beta distribution (short-dashed line) under passive (black line) and active adaptive (gray line) management. Prior belief in the colonization rate $E(c)$ was represented with a beta $(1,2)$ distribution.

actions that yield guaranteed rather than uncertain rewards (Figs. 1-4). This result is somewhat counterintuitive; one might expect that the best way to learn about colonization while managing a metapopulation is to intentionally create more learning opportunities early in a management program, sacrificing immediate benefits to improve management in the long term. However, the optimal active adaptive management strategy in this study did not support active learning; rather the best way to achieve our management objective was to take the guaranteed benefits of adding area while learning about the colonization rate by monitoring existing vacant patches. In other words, the optimal way to balance the goal of maximizing the management objective with learning was to take an action with known benefits (still allowing us to learn), rather than experimenting with a less well-understood action that could yield either greater benefits or greater losses. The optimal active adaptive strategy probably favored a precautionary approach because the value of experimentation was low: we could still learn about colonization regardless of which action was chosen.

A widespread assumption of adaptive management is that active adaptive management encourages sacrifice of benefits early in the time frame so that management is improved in the long run. However, in the environmental science and engineering literature, active learning has been shown to be both precautionary (Bar-Shalom 1981, Hauser and Possingham 2008) and experimental (Rout et al. 2009). Rout et al. (2009) found 
considerable benefit in actively learning about the growth rate of a species translocated to a new site under active adaptive management. In contrast, Hauser and Possingham (2008) compared passive and active harvest strategies for a hypothetical population and concluded that the optimal active adaptive harvest strategy was precautionary over short to medium time horizons, rather than experimental. Whether or not the optimal active adaptive management strategy is precautionary or experimental will likely depend on the model, the relative number of learning opportunities under each action, and the duration of the time horizon. Our active adaptive management strategy may have favored adding area rather than accelerating the learning process by adding patches because we ran the SDP for only $10 \mathrm{yr}$. We attempted to run our model for longer; however, we were limited by the curse of dimensionality. Our model had five state variables $(M, n, A, \alpha, \beta)$, which resulted in 10213272 states when run over $10 \mathrm{yr}$.

By simulating management of an E. editha bayensis metapopulation, we demonstrated the difference between learning about the colonization rate (both passively and actively) compared with doing nothing or repeating the same action over time. In this instance, there was little difference between passive adaptive management, active adaptive management, and consistently adding patches each time step. The three strategies were equally effective because they followed almost the same sequence of actions over time; to add patches. The only time when area was added early in the management program was under active adaptive management. The relative performance of each strategy will depend on the number and relative benefit of actions, which will depend to some extent on the slope of the extinction-area curve and the choice of true colonization rate. For example, a lower colonization rate would have made the strategy of always adding patches less effective relative to adding area. What the simulations do illustrate, however, is how managers of E. editha bayensis can adjust their actions over time based on a prior belief in the colonization rate and in response to what is learned (either passively or actively) over time. This removes the risk of committing to a suboptimal strategy (in this case consistently adding area) at the start of a management program.

As with all models, a number of important assumptions were made in our study. Although we found the optimal adaptive management strategy for two objectives, a number of other objectives could just have easily been chosen. For example, managers might seek to maximize the number of patches occupied over the duration of the management program or minimize the cost of management. By running the SDP under two management objectives, we demonstrate that adaptive strategies can be sensitive to the choice of management objective. For instance, there were many more belief states in which it was best to add patches under objective 2; that is, when the goal of management was to maximize the number of patches occupied at the end of the management program. The sensitivity of management decisions to alternative strategies is well-documented in the conservation literature (Nicholson and Possingham 2006, McCarthy and Possingham 2007). Therefore, we stress the importance of carefully considering management objectives at the start of the decision-making process. Failing to formulate clear and achievable objectives is also a major barrier to the successful implementation of adaptive management programs (Susskind et al. 2012).

All patches in the metapopulation were assumed to be identical in area and spatially implicit. This meant that we could not store individual patch areas or interpatch distances, assess the impact of corridors as a management action, or specify the location of new patches. Learning about the colonization rate for a spatially explicit mainland-island metapopulation would have added considerable complexity to our SDP. Rather than use a beta distribution to model the colonization rate, we would have required a dispersal kernel for the source patch describing the decrease in the colonization rate with distance. This kernel would have had to be updated using Bayesian statistics for all possible combinations of colonization events at every point in time. Furthermore, our SDP would have been made even more complicated if our metapopulation was a Levins model (Levins 1969) because dispersal kernels would have to be created for every possible dispersal pathway. If there are $M$ patches in a metapopulation, this equates to $2^{M}$ dispersal kernels, all of which would have to be updated for all possible combination of occupancy states at each point in time. By modeling uncertainty in our knowledge of the colonization rate using a beta distribution for a spatially implicit mainland-island metapopulation, we reduced the computational requirements substantially. We modeled belief in the colonization rate using only two state variables, $\alpha$ and $\beta$, which allowed us to update model belief relatively simply (McCarthy and Possingham 2007, Hauser and Possingham 2008, Rout et al. 2009).

Although mainland-island metapopulations receive less attention than standard Levins models, they are relatively common in natural systems (Harrison et al. 1988). There is evidence to suggest that many populations occupy mainland-island metapopulations, particularly species that exhibit extreme differences in patch size. Examples of mainland-island metapopulations have been documented for various butterflies (Shahabuddin and Terborgh 1999, Hanski and Gaggiotti 2004) and grasshoppers (Stelter et al. 1997). They can also be used to characterize weed invasion and management into sets of locations from a large external source. Learning about the colonization rate for a Levins metapopulation using our approach presents considerable challenges. If we treat vacant patches as a binomial process of independent trials and we detect a failure (that is, a patch remains empty), the beta distribution 
can be easily updated by adding the number of vacant patches to $\beta$. However, if we detect a colonization event, the number of patches that contributed to that event is unobservable, meaning the value added to $\alpha$ could be any fraction between 1 and the number of vacant patches $M-n$. One way to overcome this problem might be to model the colonization rate as a partially observable Markov decision process (POMDP; Chades et al. 2008). Adopting a POMDP approach would add additional complexity to the SDP, but constitutes an important area of future research.

While we assumed the colonization rate was poorly understood, we assumed that the number of occupied patches $n$ was known with certainty at each point in time. In other words, we assumed perfect detectability of species in patches. When conducting presence-absence surveys, the probability that an individual will be detected depends on whether it is available for detection, and given availability, its probability of detection (MacKenzie et al. 2002). An individual that is within a patch may remain undetected during surveys due to fixed variables (i.e., habitat characteristics such as topography and vegetation density) and those that vary between visits such as weather conditions and breeding activity. In reality, many species have detection probabilities well below 1 , meaning that the occupancy state of the metapopulation $n$ will not be known with certainty, as was assumed in our study. The problem of incomplete detection in discrete time Markov chain models can be solved using POMDP by assigning a belief to the uncertain state (Chades et al. 2008). Incorporating POMDP into our metapopulation model to account for imperfect detectability warrants further investigation.

Our equal cost constraint adopted from Nicol and Possingham (2010) meant that adding a new patch contributed to the same area as the current area of patches, which resulted in larger and larger patches being added to the metapopulation over time. This assumption also resulted in larger patches being added after adding to patch area earlier. Thus, there was a bias toward adding to the area of patches early in the time horizon. While this assumption is unlikely to occur in reality (some actions will cost more than others), it allowed us to directly compare the effect of each action on the management objective. Our model could be further developed to incorporate a fixed budget at each point in time and an allocation of this budget between management and monitoring (McCarthy and Possingham 2007) as well as between management actions (Ross and Pollett 2010). This may, in turn, influence the choice of management strategy (Chades et al. 2008). For simplicity, we assumed that the cost of monitoring was negligible (or equal across management options); however, assessing the occupancy status of patches can require a long-term, intensive, and costly monitoring programs.

We assumed that learning about the colonization rate was more important than learning about the extinction-area relationship. We could have expanded the framework to account for uncertainty in more than one parameter; however, this would involve adding more states to the SDP for each uncertain parameter. As already mentioned, the SDP presented here contained five state variables $(M, n, A, \alpha, \beta)$, which restricted our analysis to a small number of patches over a relatively short time horizon. Modeling uncertainty in both the extinction-area relationship and the colonization rate would not only increase the computational requirements, but would be challenging to interpret and present to decision-makers. Conducting an expected value of information analysis (EVPI) might be one way to determine which of these uncertainties should be resolved through adaptive management (Runge et al. 2011). Approximation techniques such as heuristic sampling are promising areas of research that find near-optimal solutions for complex problems with large state spaces (Nicol and Chades 2011).

\section{CONCLUSION}

We have developed a framework for managing mainland-island metapopulations when we are uncertain about the colonization rate. Given the rate and scale at which habitat fragmentation is occurring around the globe, the urgency of management intervention which is required, and the uncertainty surrounding the dynamics of these systems, developing methods that allow managers to act immediately while resolving uncertainties that impact on management decisions is crucial. While the framework presented here focuses on the management of a threatened species, it could be applied to analogous systems, such as to learn about the infection rate of a disease entering a metapopulation or to track how the true colonization rate changes through time in response to factors like climate change. A crucial area of research in conservation biology is developing frameworks that integrate modelbased decision-making with methods that resolve uncertainty within a decision-theoretic approach. This research provides a framework for learning about the uncertainty of complex processes central to the dynamics of metapopulations.

\section{ACKNOWLedgments}

This work was funded by the Australian Research Council Centre of Excellence for Environmental Decisions (CEED). C. E. Hauser was supported by the NERP Environmental Decisions Hub. We thank E. J. Milner-Gulland and Eve McDonald-Madden, who provided feedback on initial ideas.

\section{Literature Cited}

Bar-Shalom, Y. 1981. Stochastic dynamic programming: caution and probing. IEEE Transactions on Automated Control 26:1184-1195.

Bellman, R. 1957. Dynamic programming. Princeton University Press, Princeton, New Jersey, USA. 
Bormann, B. T., and A. R. Kiester. 2004. Options forestry: acting on uncertainty. Journal of Forestry 102:22-27.

Burgman, M. 2005. Risks and decisions for conservation and environmental management. Cambridge University Press, Cambridge, UK.

Burgman, M. A., D. B. Lindenmayer, and J. Elith. 2005. Managing landscapes for conservation under uncertainty. Ecology 86:2007-2017.

Chades, I., E. McDonald-Madden, M. A. McCarthy, B. Wintle, M. Linkie, and H. P. Possingham. 2008. When to stop managing or surveying cryptic threatened species. Proceedings of the National Academy of Sciences USA 105:13936-13940.

Chatfield, C. 1995. Model uncertainty, data mining and statistical inference. Journal of the Royal Statistical Society A 158:419-466.

Day, J. R., and H. P. Possingham. 1995. A stochastic metapopulation model with variability in patch size and position. Theoretical Population Biology 48:333-360.

Ehrlich, P. R. 1961. Intrinsic barriers to dispersal in checkerspot butterfly. Science 134:108-109.

Ehrlich, P. R., R. R. White, M. C. Singer, S. W. McKechnie, and L. E. Gilbert. 1975. Checkerspot butterflies: historical perspective. Science 188:221-228.

Ehrlich, P. R., D. D. Murphy, M. C. Singer, C. B. Sherwood, R. R. White, and I. L. Brown. 1980. Extinction, reduction, stability and increase: the responses of checkerspot butterfly (Euphydryas) populations to the Californian drought. Oecologia 46:101-105.

Etienne, R. S., and J. A. P. Heesterbeek. 2001. Rules of thumb for conservation of metapopulations based on a stochastic winking-patch model. American Naturalist 158:389-407.

Frisch, D., K. Cottenie, A. Badosa, and A. J. Green. 2012. Strong spatial influence on colonization rates in a pioneer zooplankton metacommunity. PLoS ONE 7:e40205.

Gerber, L. R., M. Beger, M. A. McCarthy, and H. P. Possingham. 2005. A theory for optimal monitoring of marine reserves. Ecology Letters 8:829-837.

Gilpin, M. E., and J. M. Diamond. 1976. Calculation of immigration and extinction curves from species-area-distance relation. Proceedings of the National Academy of Sciences USA 73:4130-4134.

Grantham, H. S., K. A. Wilson, A. Moilanen, T. Rebelo, and H. P. Possingham. 2009. Delaying conservation actions for improved knowledge: how long should we wait? Ecology Letters 12:293-301.

Hanski, I. 1994. A practical model of metapopulation dynamics. Journal of Animal Ecology 63:151-162.

Hanski, I. A., and O. E. Gaggiotti. 2004. Ecology genetics and evolution of metapopulations. Academic Press, London, UK.

Harrison, S., D. D. Murphy, and P. R. Ehrlich. 1988. Distribution of the bay checkerspot butterly Euphydryas editha bayensis: evidence for a metapopulation model. American Naturalist 132:360-382.

Hauser, C. E., and H. P. Possingham. 2008. Experimental or precautionary? Adaptive management over a range of time horizons. Journal of Applied Ecology 45:72-81.

Holling, C. S. 1978. Adaptive environmental assessment and management. John Wiley and Sons, London, UK

Isaak, D. J., R. F. Thurow, B. E. Rieman, and J. B. Dunham. 2007. Chinook salmon use of spawning patches: relative roles of habitat quality, size, and connectivity. Ecological Applications 17:352-364.

Launer, A. E., and D. D. Murphy. 1994. Umbrella species and the conservation of habitat fragments: a case study of a threatened butterfly and vanishing grassland ecosystem. Biological Conservation 69:145-153.
Levins, R. 1969. Some demographic and genetic consequences of environmental heterogeneity for biological control. Bulletin of the Entomological Society of America 15:237-240.

Lubow, B. C. 1996. Optimal translocation strategies for enhancing stochastic metapopulation viability. Ecological Applications 6:1268-1280.

Ludwig, D., R. Hilborn, and C. Walters. 1993. Uncertainty, resource exploitation, and conservation: lessons from history. Science 260:17-18.

MacKenzie, D. I., J. D. Nichols, G. B. Lachman, S. Droege, J. A. Royle, and C. A. Langtimm. 2002. Estimating site occupancy rates when detection probabilities are less than one. Ecology 83:2248-2255.

Maguire, L. A. 1986. Using decision-analysis to manage endangered species populations. Journal of Environmental Management 22:345-360.

Mangel, M., and C. W. Clark. 2000. Dynamic state variable models in ecology: methods and applications. Oxford University Press, New York, New York, USA.

Marescot, L., G. Chapron, I. Chades, P. L. Fackler, C. Duchamp, E. Marboutin, and O. Gimenez. 2013. Complex decisions made simple: a primer on stochastic dynamic programming. Methods in Ecology and Evolution 4:872-884.

McCarthy, M. A., and H. P. Possingham. 2007. Active adaptive management for conservation. Conservation Biology 21:956-963.

McDonald-Madden, E., W. J. M. Probert, C. E. Hauser, M. C. Runge, H. P. Possingham, M. E. Jones, J. L. Moore, T. M. Rout, P. A. Vesk, and B. A. Wintle. 2010. Active adaptive conservation of threatened species in the face of uncertainty. Ecological Applications 20:1476-1489.

Moilanen, A., and M. Cabeza. 2002. Single-species dynamic site selection. Ecological Applications 12:913-926.

Moore, A. L., and M. A. McCarthy. 2010. On valuing information in adaptive-management models. Conservation Biology 24:984-993.

Murphy, D. D. 1984. Butterflies and their nectar plants: the role of the checkerspot butterfly Euphydryas editha as a pollen vector. Oikos 43:113-117.

Murphy, D. D., K. E. Freas, and S. B. Weiss. 1990. An environment-metapopulation approach to population viability analysis for a threatened invertebrate. Conservation Biology 4:41-51.

Nichols, J. D., F. A. Johnson, and B. K. Williams. 1995. Managing North American waterfowl in the face of uncertainty. Annual Review of Ecology and Systematics 26:177-199.

Nicholson, E., and H. P. Possingham. 2006. Objectives for multiple-species conservation planning. Conservation Biology 20:871-881.

Nicol, S., and I. Chades. 2011. Beyond stochastic dynamic programming: a heuristic sampling method for optimizing conservation decisions in very large state spaces. Methods in Ecology and Evolution 2:221-228.

Nicol, S. C., and H. P. Possingham. 2010. Should metapopulation restoration strategies increase patch area or number of patches? Ecological Applications 20:566-581.

Ovaskainen, O., and I. Hanski. 2004. Metapopulation dynamics in a highly fragmented landscape. Pages 73-103 in I. Hanski, and O. E. Gaggiotti, editors. Ecology, genetics and evolution of metapopulations. Elsevier, Amsterdam, Netherlands.

Parma, A. M., et al. 1998. What can adaptive management do for our fish, forests, food and biodiversity? Integrative Biology, Issues, News, and Reviews 1:16-26.

Possingham, H. 1996. Decision theory and biodiversity management: how to manage a metapopulation. Pages 391-398 in R. B. Floyd, A. W. Sheppard, and P. J. De 
Barro, editors. Frontiers of population ecology. CSIRO Publishing, Melbourne, Australia.

Possingham, H., S. J. Andelman, B. R. Noon, S. Trombulak, and H. R. Pulliam. 2001. Making smart conservation decisions. Pages 225-244 in M. E. Soule, and G. H. Orians, editors. Conservation biology: research priorities for the next decade. Island Press, Washington, D.C., USA.

Preston, K. L., R. A. Redak, M. F. Allen, and J. T. Rotenberry 2012. Changing distribution patterns of an endangered butterfly: linking local extinction patterns and variable habitat relationships. Biological Conservation 152:280-290.

R Development Core Team. 2014. R: a language and environment for statistical computing. R Foundation for Statistical Computing, Vienna, Austria. Available at: www.r-project.org

Regan, H. M., M. Colyvan, and M. A. Burgman. 2002. A taxonomy and treatment of uncertainty for ecology and conservation biology. Ecological Applications 12:618-628.

Ross, J. V., and P. K. Pollett. 2010. Simple rules for ranking and optimally managing metapopulations. Ecological Modelling 221:2515-2520.

Ross, J. V., D. J. Sirl, P. K. Pollett, and H. P. Possingham. 2008. Metapopulation persistence in a dynamic landscape more habitat or better stewardship? Ecological Applications 18:590-598.

Rout, T. M., C. E. Hauser, and H. P. Possingham. 2009. Optimal adaptive management for the translocation of a threatened species. Ecological Applications 19:515-526.

Runge, M. C., and F. A. Johnson. 2002. The importance of functional form in optimal control solutions of problems in population dynamics. Ecology 83:1357-1371.

Runge, M. C., S. J. Converse, and J. E. Lyons. 2011. Which uncertainty? Using expert elicitation and expected value of information to design an adaptive program. Biological Conservation 144:1214-1223.

Schultz, C. B., and E. E. Crone. 2005. Patch size and connectivity thresholds for butterfly habitat restoration. Conservation Biology 19:887-896.
Shahabuddin, G., and J. W. Terborgh. 1999. Frugivorous butterflies in Venezuelan forest fragments: abundance, diversity and the effects of isolation. Journal of Tropical Ecology 15:703-722.

Shea, K., H. P. Possingham, W. W. Murdoch, and R. Roush. 2002. Active adaptive management in insect pest and weed control: intervention with a plan for learning. Ecological Applications 12:927-936.

Smith, A. D. M., and C. J. Walters. 1981. Adaptive management of stock recruitment systems. Canadian Journal of Fisheries and Aquatic Sciences 38:690-703.

Stelter, C., M. Reich, V. Grimm, and C. Wissel. 1997. Modelling persistence in dynamic landscapes: lessons from a metapopulation of the grasshopper Bryodema tuberculata. Journal of Animal Ecology 66:508-518.

Susskind, L., A. E. Camacho, and T. Schenk. 2012. A critical assessment of collaborative adaptive management in practice. Journal of Applied Ecology 49:47-51.

United States Federal Register. 1987. 52 FR 35366. U.S. National Archives \& Records Administration, College Park, Maryland, USA.

Walters, C. J. 1986. Adaptive management of renewable resources. Macmillan, New York, New York, USA.

Walters, C. J., and R. Hilborn. 1976. Adaptive control of fishing systems. Journal of the Fisheries Research Board of Canada 33:145-159.

Walters, C., L. Gunderson, and C. S. Holling. 1992. Experimental policies for water management in the everglades. Ecological Applications 2:189-202.

Westphal, M. I., M. Pickett, W. M. Getz, and H. P. Possingham. 2003. The use of stochastic dynamic programming in optimal landscape reconstruction for metapopulations. Ecological Applications 13:543-555.

Williams, B. K. 1996. Adaptive optimization and the harvest of biological populations. Mathematical Biosciences 136:1-20.

Williams, B. K., and F. A. Johnson. 1995. Adaptive management and the regulation of waterfowl harvests. Wildlife Society Bulletin 23:430-436.

\section{SUPPORTING INFORMATION}

Additional supporting information may be found in the online version of this article at http://onlinelibrary.wiley.com/ doi/10.1890/14-2430.1/suppinfo 